\title{
THE
}

UNIVERSITY

University of Rhode Island

OF RHODE ISLAND

DigitalCommons@URI

10-1-1986

\section{Nature of Quantum Chaos in Spin Systems}

Gerhard Müller

University of Rhode Island, gmuller@uri.edu

Follow this and additional works at: https://digitalcommons.uri.edu/phys_facpubs

Terms of Use

All rights reserved under copyright.

\section{Citation/Publisher Attribution}

Gerhard Müller. Nature of quantum chaos in spin systems. Phys. Rev. A 34 (1986), 3345-3355.

Available at: http://dx.doi.org/10.1103/PhysRevA.34.3345

This Article is brought to you for free and open access by the Physics at DigitalCommons@URI. It has been accepted for inclusion in Physics Faculty Publications by an authorized administrator of DigitalCommons@URI. For more information, please contact digitalcommons-group@uri.edu. 


\title{
Nature of quantum chaos in spin systems \\ Gerhard Müller \\ Department of Physics, University of Rhode Island, Kingston, Rhode Island 02881
}

(Received 27 January 1986)

\begin{abstract}
A novel concept for quantum chaos in spin systems is proposed which differs distinctively from concepts currently in use. It is argued (a) that the definition of quantum chaos cannot be based on the correspondence principle and (b) that quantum chaos is not equivalent to statistical behavior. Quantum chaos in spin systems implies the existence of a new type of spectrum consisting of excitations which do not form regular patterns with a multiparameter continuum structure in the thermodynamic limit, thus removing the constraint imposed by van Hove singularities that time-dependent correlation functions for pure quantum states cannot decay more rapidly than as powers of $t$. The irregular spectrum is caused by strong level repulsion resulting from the lack of a sufficient number of conservation laws in nonintegrable quantum many-body systems. The similarities and differences between quantum and classical chaos and, more generally, between quantum (non)integrability and classical (non)integrability are discussed in detail.
\end{abstract}

\section{INTRODUCTION}

The study of quantum chaos has been an area of intensive research activity in recent years. However, no generally accepted agreement has been reached on its exact nature. The two major points of view may be paraphrased as follows: ${ }^{1}$

(i) Quantum chaos has its manifestations in spectral irregularities and related phenomena observable in quantum systems with few degrees of freedom whose classical counterparts are dynamically nonintegrable.

(ii) Quantum chaos is that property which causes a quantum system to behave statistically in the sense that the time evolution of a dynamical variable approaches an equilibrium value and that this value is the one predicted by quantum statistical mechanics.

From the following critique of the two points of view, embedded in a discussion of the various concepts of integrability for classical and quantum systems, a new definition for quantum chaos will naturally emerge. Its characteristic properties and implications for the time evolution of quantum systems will be outlined and its relation to both the concept of classical deterministic chaos and the concept of nonintegrability in classical statistical models will be established.

For the sake of economy in the formalism and precision in the language, the entire presentation will focus on systems of interacting (quantum and classical) spins. However, many of the concepts and conclusions are expected to translate into corresponding concepts and conclusions for dynamical systems of a different nature.

\section{INTEGRABILITY IN CLASSICAL SPIN SYSTEMS}

There are two concepts of integrability in use for classical spin systems.

Dynamical integrability: A classical spin system is dynamically integrable (completely integrable, separable) if there exist $N$ distinct integrals of the motion in involution, where $N$ is the number of degrees of freedom. ${ }^{2,3}$

Thermodynamic integrability: A classical spin system is thermodynamically integrable (exactly solvable) if it is possible to determine its partition function exactly. ${ }^{4,5}$ The following discussion of these two concepts of integrability is intended to clarify their relationship to the concept of integrability for quantum systems to be discussed in Sec. III.

\section{A. Dynamical integrability}

Consider a system of $N$ localized classical threecomponent spins $\mathbf{S}_{l}, l=1,2, \ldots, N$ specified by some interaction Hamiltonian $\mathscr{H}\left[\mathbf{S}_{1}, \mathbf{S}_{2}, \ldots, \mathbf{S}_{N}\right]$. Its time evolution is determined by the Hamilton equation of motion

$$
\frac{d}{d t} \mathbf{S}_{l}=\left\{\mathscr{H}, \mathbf{S}_{l}\right\} \text {, }
$$

where the right-hand side is the Poisson bracket defined as

$$
\{A, B\}=\sum_{l}\left(\frac{\partial A}{\partial p_{l}} \frac{\partial B}{\partial q_{l}}-\frac{\partial B}{\partial p_{l}} \frac{\partial A}{\partial q_{l}}\right)
$$

with respect to a set of canonical variables $p_{l}$ and $q_{l}$. The Poisson brackets for classical spin variables, i.e., the sympletic structure for classical spin dynamics, can be constructed by requiring that the resulting Hamilton equation of motion (2.1) is consistent with the Heisenberg equation of motion

$$
\frac{d}{d t} \mathbf{S}_{l}=i\left[\mathscr{H}, \mathbf{S}_{l}\right]
$$

for $\mathbf{S}_{l}$ interpreted as quantum spin operators, whose commutation relations

$$
\left[S_{l}^{\alpha}, S^{\beta}\right]=i \delta_{l l^{\prime}} \sum_{\gamma} \epsilon^{\alpha \beta \gamma} S_{l}^{\gamma}
$$

are part of their intrinsic properties. Here $\epsilon^{\alpha \beta \gamma}$ is the 
Levi-Cività symbol and $\delta_{l l^{\prime}}$ the Kronecker symbol. For the classical spins this leads to the result ${ }^{6}$

$$
\left\{S_{l}^{\alpha}, S_{l^{\prime}}^{\beta}\right\}=-\delta_{l l^{\prime}} \sum_{\gamma} \epsilon^{\alpha \beta \gamma} S_{l}^{\gamma} .
$$

If the classical spins $\mathbf{S}_{l}$ are expressed in terms of polar coordinates as

$$
\mathbf{S}_{l}=s\left(\sin \theta_{l} \cos \phi_{l}, \sin \theta_{l}, \sin \phi_{l}, \cos \theta_{l}\right)
$$

then a set of canonical variables which satisfy the relation

$$
\left\{p_{l}, q_{l^{\prime}}\right\}=\delta_{l l^{\prime}}, \quad\left\{p_{l}, p_{l^{\prime}}\right\}=\left\{q_{l}, q_{l^{\prime}}\right\}=0
$$

are given by

$$
p_{l}=\cos \theta_{l}, \quad q_{l}=\phi_{l} .
$$

Thus a system of $N$ classical spins specified by an energy functional $\mathscr{H}\left[\mathbf{S}_{1}, \mathbf{S}_{2}, \ldots, \mathbf{S}_{N}\right]$ represents an autonomous Hamiltonian system of $N$ degrees of freedom. The system is dynamically integrable if there exist $N$ distinct integrals of the motion in involution

$$
J_{k}\left[\mathbf{S}_{1}, \mathbf{S}_{2}, \ldots, \mathbf{S}_{N}\right]=\mathrm{const}, \quad k=1,2, \ldots, N
$$

with

$$
\begin{aligned}
& \left\{\mathscr{H}, J_{k}\right\}=0, \quad k=1,2, \ldots, N, \\
& \left\{J_{k}, J_{k^{\prime}}\right\}=0, \quad k, k^{\prime}=1,2, \ldots, N .
\end{aligned}
$$

The existence of $N$ such integrals of the motion confines any individual trajectory in the $2 N$-dimensional phase space $^{7}$ to the intersection of the $N(2 N-1)$-dimensional hypersurfaces $J_{k}=$ const, $k=1,2, \ldots, N$. The resulting $N$-dimensional hypersurface is called an invariant torus, because it is possible to choose the $J_{k}$ such that their numerical values are determined by $N$ action integrals of the form

$$
2 \pi J_{k}=\oint_{C_{k}} p_{k} d q_{k}, k=1,2, \ldots, N,
$$

where the $C_{k}$ are $N$ topologically independent closed paths. For an autonomous system, the Hamiltonian itself is an integral of the motion reflecting (in the present application but not in general) the conservation of the total energy. Equivalent to this fact is the property that the energy functional can be expressed in terms of the $N$ action variables $J_{k}$ alone

$$
\mathscr{H}\left[\mathbf{S}_{1}, \mathbf{S}_{2}, \ldots, \mathbf{S}_{N}\right]=\mathscr{H}^{\prime}\left[J_{1}, J_{2}, \ldots, J_{N}\right] .
$$

This is the classical spin Hamiltonian in the action-angle representation, leading to $2 N$ canonical equations which have trivial solutions involving at most $N$ distinct frequencies

$$
\omega_{k}=\frac{\partial H^{\prime}}{\partial J_{k}}, k=1,2, \ldots, N
$$

in the time evolution. The consequence is that the time evolution of the spin variables is characterized by a discrete spectrum. ${ }^{8}$

If the number of distinct integrals of the motion is $n<N$, the constraints imposed on the trajectories in $2 N$ dimensional phase space are more relaxed compared to the integrable case. The foliation of the entire phase space by invariant tori is at least partially destroyed, the consequence being that there exist, in addition to regular trajectories, which are still confined to $\mathrm{N}$-dimensional invariant tori, also trajectories representing phase points moving over hypersurfaces of dimensionality larger than $N$. Such trajectories are chaotic in character. The Fourier spectrum of a chaotic trajectory is continuous as opposed to the discrete spectrum of a regular trajectory.

For a brief illustration of the concept of dynamical integrability, consider a chain of $N$ classical spins coupled to one another by an isotropic Heisenberg exchange interaction

$$
\mathscr{H}=-J \sum_{l=1}^{N-1} \mathbf{S}_{l} \cdot \mathbf{S}_{l+1}
$$

There exist three obvious integrals of the motion which are in involution.

(1) The total energy: $E=\mathscr{H}\left[\mathbf{S}_{1}, \mathbf{S}_{2}, \ldots, \mathbf{S}_{N}\right]$.

(2) The $z$-component of the total spin: $S_{T}^{z}=\sum^{N} S_{l}^{z}$.

(3) The total spin: $S_{T}=\left[\left(S_{T}^{x}\right)^{2}+\left(S_{T}^{y}\right)^{2}+\left(S_{T}^{z}\right)^{l}\right]^{1 / 2}$.

Note that the three Cartesian components of the total spin, $S_{T}^{x}, S_{T}^{y}, S_{T}^{z}$, cannot simultaneously be used as action variables although they are simultaneously conserved, because they violate the involution condition (2.10b). We conclude that the Heisenberg spin cluster is dynamically integrable at least for $N=2$ and 3. In the presence of a uniaxial exchange anisotropy, the third conservation law is destroyed: the system is still dynamically integrable for $N=2$ but not necessarily for $N \geq 3 .{ }^{9}$ Chaotic behavior may even be found in autonous two-spin systems if the energy functional $\mathscr{H}\left[\mathbf{S}_{1}, \mathbf{S}_{2}\right]$ is sufficiently asymmetric. There exists numerical evidence that the following two models are dynamically nonintegrable:

(i) The $X Y$ model with single-ion anisotropy, ${ }^{10}$

$$
\mathscr{H}=-J\left(S_{1}^{x} S_{2}^{x}+S_{1}^{y} S_{2}^{y}\right)-\frac{1}{2} A\left(S_{1}^{x} S_{1}^{x}+S_{2}^{x} S_{2}^{x}\right) .
$$

(ii) The Ising model in a transverse field, ${ }^{11,12}$

$$
\mathscr{H}=-J S_{1}^{z} S_{2}^{z}-h\left(S_{1}^{x}+S_{2}^{x}\right) .
$$

By contrast, any autonomous one-spin system is integrable according to our preceding discussion. However, if a single spin is coupled to a time-dependent field, integrability is no longer guaranteed. Frahm and Mikeska ${ }^{13}$ indeed observed chaotic trajectories in the nonautonomous ${ }^{14}$ onespin system

$$
\mathscr{H}=A\left(S^{z}\right)^{2}-h \cos (\omega t) S^{x} .
$$

\section{B. Thermodynamic integrability}

For a classical spin system consisting of $N$ spins, the partition function can be expressed as an $N$-fold integral

$\left.Z_{N}=\int d \mathbf{S}_{1} \int d \mathbf{S}_{2} \cdots \int d \mathbf{S}_{N} e^{-\beta \not r} \mathbf{S}_{1}, \mathbf{S}_{2}, \ldots, \mathbf{S}_{N}\right]$.

As long as $N$ is finite, $Z_{N}$ is a well-behaved, analytic function. Unlike the integrals of the Hamilton equation of motion (2.1), $Z_{N}$ is an integral over a compact manifold, i.e., does not involve any asymptotic behavior which 
could give rise to surprises. It is therefore legitimate to call finite classical spin systems thermodynamically integrable even though the execution of the integral (2.18) may pose insurmountable technical problems for moderately large $N$. Hence only in the limit $N \rightarrow \infty$ can thermodynamic integrability be a meaningful attribute of classical spin systems. In the thermodynamic limit, the analytic properties of the partition function undergo a qualitative change, and there exists no general theory from which they can be inferred. Nevertheless, we have knowledge of a large number of interacting classical spin models which are thermodynamically integrable in the limit $N \rightarrow \infty$ They include, for example, all one-dimensional (1D) models with nearest-neighbor interaction. ${ }^{15}$ For these systems, thermodynamic integrability is established quite generally by the transfer-operator technique.

Consider a general 1D spin Hamiltonian of the form

$$
\mathscr{H}=\sum_{l=1}^{N} U\left(\mathbf{S}_{l}, \mathbf{S}_{l+1}\right)
$$

with periodic boundary conditions imposed. The partition function (2.18) can then be expressed as the trace of the $N^{\text {th }}$ iterate of the integral operator whose kernel has the form

$$
L\left(\mathbf{S}_{1}, \mathbf{S}_{2}\right)=e^{-\beta U\left(\mathbf{S}_{1}, \mathbf{S}_{2}\right)} .
$$

This is the transfer operator. In terms of its eigenvalues $\lambda_{n}$, which are determined from the equation

$$
\int d \mathbf{S}_{2} L\left(\mathbf{S}_{1}, \mathbf{S}_{2}\right) \psi_{n}\left(\mathbf{S}_{2}\right)=\lambda_{n} \psi_{n}\left(\mathbf{S}_{1}\right), \quad n=0,1,2, \ldots,
$$

the partition function can be rewritten as

$$
Z_{N}=\sum_{n=0}^{\infty} \lambda_{n}^{N}=\lambda_{0}^{N}\left[1+\sum_{n=1}^{\infty}\left(\lambda_{n} / \lambda_{0}\right)^{N}\right)
$$

In the thermodynamic limit $N \rightarrow \infty$, the Gibbs free energy per site is then determined by $\lambda_{0}$, the largest eigenvalue of $L$, alone,

$$
\mathbf{G}=-k_{B} T \lim _{N \rightarrow \infty}\left(N^{-1} \ln Z_{N)}=-k_{B} T \ln \lambda_{0}\right.
$$

Analytic solutions are known for a large class of 1D models. ${ }^{16}$

In higher dimensions $(D>1)$, thermodynamically integrable classical spin systems are probably the exception rather than the rule. Even for 2D systems, transfer operators are at best infinite matrices or infinite-dimensional integral operators, whose general properties are not known and are therefore prone to surprises. Nevertheless, 2D classical spin models which are thermodynamically integrable do exist. The Ising model is the prototype, and a growing number of further examples appear in the literature. $^{5}$

Thermodynamic integrability of an infinite classical spin system implies the existence of an infinite set of conservation laws, which are at the basis of any method to diagonalize the transfer operator. ${ }^{5,17}$ The available results for a variety of exactly solved models show that the spectrum of their transfer operator can be expressed as com- posites of quasiparticle excitations. ${ }^{5}$ The critical behavior of these exactly solved models can then be analyzed in terms of the quasiparticle spectrum in the vicinity of the largest eigenvalue $\lambda_{0}$, which alone already determines the free energy. Near a critical point, the gap between the maximum eigenvalue and the upper threshold of the remaining spectrum defines a correlation length. ${ }^{18}$ As the critical point is approached, the gap goes to zero and the correlation length diverges. Because of the quasiparticle nature of the spectrum, an infinite quasidegeneracy of eigenvalues builds up at $\lambda_{0}$. This quasidegeneracy is a crucial prerequisite for the standard critical behavior at a continuous phase transition with all the well-known scaling properties.

The preceding observations suggest that in thermodynamically nonintegrable classical spin models the quasiparticle structure is not a generic property of the spectrum of the transfer operator, in analogy to the fact discussed in Sec. II A that in dynamically nonintegrable classical spin systems the foliation of the phase space by invariant tori is at least partially destroyed. In both situations, nonintegrability is attributable to the lack of a sufficient number of conservation laws. In the dynamical system it results in the existence of chaotic trajectories, whereas in the thermodynamic system it is likely to lead to the phenomenon of "level repulsion" in the spectrum of the transfer operator. Whether this latter property is manifest at the edge of the spectrum where it could affect the scaling properties of a thermodynamically nonintegrable model in the vicinity of a continuous phase transition is a very important unsolved question. ${ }^{17}$ In Sec. III B it will be argued that thermodynamic nonintegrability is related to the question of quantum chaos in many-body systems.

\section{INTEGRABILITY IN QUANTUM SPIN SYSTEMS}

In contrast to classical spin systems, there exists only a single concept of integrability for quantum spin systems which pertains to both its thermodynamic and its dynamical properties. Both the thermodynamic properties of the quantum system and the time evolution of any dynamical variable depend on the structure of the Hamiltonian operator and the spin-commutation relations. In classical spin systems, on the other hand, the thermodynamic properties are determined by the energy functional $\mathscr{H}\left[\mathbf{S}_{1}, \mathbf{S}_{2}, \ldots, \mathbf{S}_{N}\right]$ alone, i.e., by what is commonly but somewhat imprecisely called the "classical spin Hamiltonian;" the dynamical properties further depend on the specification of a symplectic structure, i.e., the specification of Poisson brackets for classical spin variables or the expression of $\mathscr{H}\left[\mathbf{S}_{1}, \mathbf{S}_{2}, \ldots, \mathbf{S}_{N}\right]$ in terms of canonical variables.

Model systems containing a finite number of interacting quantum spins are always integrable. The underlying Hilbert space has a finite dimensionality $(2 s+1)^{N}$, where $N$ is the number of spins, each spin with the same quantum number $s$. The time evolution of any physical quantity is then either periodic or multiperiodic (quasiperiodic). Effects of nonintegrability can therefore be expected only in either one of the following two limits: 
Classical limit: $N$ finite, $s \rightarrow \infty$.

Thermodynamic limit: $s$ finite, $N \rightarrow \infty$.

It will be argued that nonintegrability effects observed in small quantum-spin clusters for large $s$ are precursors of classical dynamical chaos and that nonintegrability effects observed in quantum-spin systems for large $N$ are precursors of quantum chaos.

\section{A. Classical limit: $N$ finite, $s \rightarrow \infty$}

The intensive search of recent years for realizations of quantum chaos has focused almost exclusively on quantum systems with few degrees of freedom whose classical counterparts are known to be dynamically nonintegrable. ${ }^{1}$ To this category belong the nonintegrable spin-cluster models discussed in Sec. II A. Typical observations on such small spin clusters have been the following. ${ }^{19}$

The quantum spectrum, which is always discrete for finite-spin systems, was found to have a qualitatively different structure in different energy intervals, depending on whether the classical counterpart has predominantly regular or predominantly chaotic trajectories in that interval. ${ }^{9,12,13,20}$ In the regular parts of the spectrum, the energy eigenvalues have a tendency to cluster into sets of near- ly equidistant levels. No such patterns of regularity can be discerned in those parts of the spectrum (the irregular spectrum) where choatic trajectories are predominant in the classical system. If the spectrum is plotted as a function of some parameter which controls the integrability of the model system, ${ }^{21}$ level repulsion in the regular parts of the spectrum makes its appearance in the form of very nearly avoided crossings with extremely narrow gaps. In the irregular parts of the spectrum, on the other hand, the levels tend to repel one another very strongly. Here, the regions of avoided crossings are very broad and overlap. ${ }^{20}$ The consequence is that any complete specification and classification of energy levels in terms of the quantum numbers assigned to them in one or the other integrable limit of the model loses its meaning as they are traced sufficiently far through the mesh of avoided crossings.

It is tempting to identify this "level turbulence" in the spectral properties of small spin clusters as one of the characteristic features of quantum chaos. However, we should draw our attention to the fact that, in the extreme classical limit $s \rightarrow \infty$, the observed dramatic differences between regular and irregular parts of the spectrum are bound to fade away, at least on that energy scale for which $J s^{2}$ is kept finite as $s \rightarrow \infty$. In that limit, the level distribution function for the quantum spin cluster connects to the energy density of its classical counterpart,

$$
D(\epsilon)=\frac{1}{(4 \pi)^{N}} \int d \mathbf{S}_{1} \int d \mathbf{S}_{2} \cdots \int d \mathbf{S}_{N} \delta\left(\epsilon-\mathscr{H}\left[\mathbf{S}_{1}, \mathbf{S}_{2}, \ldots, \mathbf{S}_{N}\right]\right)
$$

which is a well-behaved piecewise smooth function irrespective of whether the classical spin cluster is dynamically integrable or nonintegrable. True chaos can only be found in quantities which relate to individual trajectories of the classical spin clusters or, more generally, in quantities which depend on the symplectic structure of the classical model, not just on its energy functional.

Feingold, Moiseyev, and Peres ${ }^{12}$ studied the matrix elements of simple dynamical variables for the autonomous two-spin system (2.16), which is dynamically nonintegrable in the classical limit. They found a striking difference in the role played by selection rules in the regular and irregular parts of the spectrum, respectively. In the regular spectrum a substantial fraction of matrix elements is very close to zero as if subject to some hidden selection rules. In matrix elements between states of the irregular spectrum this effect is conspicuously absent.

The most direct link between an individual trajectory of the classical spin cluster and any property of its quantum counterpart is established by the time evolution of Wigner distributions. This is what Frahm and Mikeska ${ }^{13}$ effectively studied for the nonautonomous one-spin system (2.17), which is dynamically nonintegrable in the classical limit. They found that the width of a Wigner distribution in the semiclassical regime $(s>1)$ increases roughly linearly with time $(\propto t / \sqrt{s})$ for initial conditions which correspond to a regular trajectory in the classical limit. This effect is attributable to quantum uncertainty; it disappears in the classical limit. However, if the initial conditions correspond to a chaotic trajectory in the classical limit, the width increases exponentially in time. Here, the (relatively mild) quantum uncertainty is overwhelmed by the much stronger precursors of classical dynamical chaos. Even though these results do not represent true long-time asymptotic properties for finite $s$, they nevertheless reflect the well-known property of classical dynamical systems that nearby trajectories separate (roughly) linearly in time, whereas nearby chaotic trajectories separate exponentially in time. True chaos is a long-time asymptotic property of a dynamical system which no quantum spin cluster can possibly exhibit, however, closely it approaches the classical limit. ${ }^{22}$ It seems therefore more appropriate to name these effects of nonintegrability precursors of classical dynamical chaos rather than quantum chaos. ${ }^{23}$

\section{B. Thermodynamic limit: $s$ finite, $N \rightarrow \infty$}

Dynamical properties of infinite $(N \rightarrow \infty)$ quantum spin systems are in general highly nontrivial even for integrable models. There indeed exist a number of 1D models of interacting quantum spins which are integrable. In some cases their dynamical properties have been analyzed in great detail. Integrable 1D quantum-spin models include, most prominently, the class of Betheansatz-solvable models such as the 1D $s=\frac{1}{2}$ Heisenberg model $^{24}$ 


$$
\mathscr{H}=-J \sum_{l=1}^{N} \mathbf{S}_{l} \cdot \mathbf{S}_{l+1}
$$

and some of its generalizations, such as the 1D $s=\frac{1}{2} X X Z$ model $^{25}$

$$
\mathscr{H}=-J \sum_{l=1}^{N}\left(S_{l}^{x} S_{l+1}^{x}+S S_{l+1}^{y}+\Delta S_{l}^{z} S_{l+1}^{z}\right)
$$

and the 1D spin-1 bilinear-biquadratic model ${ }^{26}$

$$
\mathscr{H}=-J \sum_{l=1}^{N}\left[\mathbf{S}_{l} \cdot \mathbf{S}_{l+1}-\left(\mathbf{S}_{l} \cdot \mathbf{S}_{l+1}\right)^{2}\right] .
$$

Quantum integrability depends, like classical dynamical integrability, on the existence of a sufficient number of conservation laws. The complete set of quantum numbers in integrable quantum-Hamiltonian systems plays a role similar to the one played by the complete set of action variables in integrable classical Hamiltonian systems.

The well-established fact that classical deterministic chaos is a property of nonintegrable classical dynamical systems as discussed in Sec. II A strongly suggests or seems to dictate almost that quantum chaos is a property of nonintegrable quantum many-body systems. Therefore any serious attempt to discern the hallmarks of quantum chaos must take account of the exactly known properties of integrable quantum many-body models and identify those properties which result as a consequence of integrability. This strategy had its counterpart in classical dynamical systems, where the foliation of the entire phase space by invariant tori was recognized as an implication of dynamical integrability, and where all the indicators of classical deterministic chaos were recognized to imply that this pattern is at least partially destroyed.

Van Kampen ${ }^{22}$ proposed that quantum chaos is that property which causes a quantum system to behave statistically in the sense that the time evolution of a dynamical variable approaches an equilibrium value and that this value is the one predicted by quantum statistical mechanics. Obviously, finite quantum-spin models do not behave statistically: the time evolution of any dynamical variable is, in general, multiperiodic. The thermodynamic limit $(N \rightarrow \infty)$ is therefore a precondition for statistical behavior as much as it is a precondition for nonintegrability.

The test of statistical behavior for any given infinite quantum-spin model is, in general, an extremely difficult task. In practice it can be carried out only for rather special situations and, with some rigor, only for the class of integrable models. In the context of equilibrium statistical mechanics, statistical behavior can be tested by studying the long-time asymptotic behavior of time-dependent correlation functions. Consider, for example, the autocorrelation function of a quantum-spin model specified by a Hamiltonian $\mathscr{H}$. For a pure quantum state $|\psi\rangle$ this function is formally expressed as

$$
\left\langle S_{l}^{\mu}(t) S_{Y}^{\mu}\right\rangle=\left\langle\psi\left|e^{i \mathscr{H} t} S_{l}^{\mu} e^{-i \mathscr{H} t} S_{Y}^{\mu}\right| \psi\right\rangle .
$$

The criterion for statistical behavior of the autocorrelation function of a given quantum-spin model can then be formulated as the following long-time asymptotic property:

$$
\lim _{t \rightarrow \infty}\left\langle S_{l}^{\mu}(t) S_{l}^{\mu}\right\rangle=\left\langle S_{l}^{\mu}\right\rangle^{2}
$$

For this admittedly quite special situation, the question whether a given many-body quantum system behaves statistically can be examined on a rigorous basis for certain 1D quantum spin models whose time-dependent correlation functions have been analyzed in the thermodynamic limit. Needless to say, all such results are restricted to integrable models. It turns out that, apart from some anomalies which are of no serious concern, ${ }^{27,28}$ none of the available exact results for the long-time asymptotic behavior of time-dependent correlation functions of $1 D$ quantum spin models is in conflict with statistical behavior. Examples will be presented below. The same conclusion was reached, albeit on a much less rigorous basis, from numerical integrations of the Schrödinger equation for various dynamical variables and initial conditions of a given quantum-spin model. ${ }^{29}$

A property of dynamic correlation functions for infinite quantum-spin systems which is likely to be more sensitive to the integrability or nonintegrability of the underlying model than the property of statistical behavior is the general structure of its excitation spectrum. Characteristic for all quantum-spin models which are integrable in the thermodynamic limit, by means of the Bethe ansatz or related techniques, is that the entire excitation spectrum is composed of multiparameter continua. ${ }^{30}$ These are structures with well-defined boundaries, which give rise to power-law or logarithmic van Hove singularities in the corresponding densities of states. Except for the unlikely event ${ }^{31}$ that these singularities are wiped out by the effects of matrix elements, they make their appearance also in the frequency-dependent autocorrelation functions

$$
\phi_{\mu \mu}(\omega)=\int_{-\infty}^{+\infty} d t e^{i \omega t}\left\langle S_{l}^{\mu}(t) S_{Y}^{\mu}\right\rangle
$$

or any other frequency-dependent quantity. If this is the case, it imposes the constraint that the corresponding time-dependent autocorrelation functions $\left\langle S_{l}^{\mu}(t) S_{l}^{\mu}\right\rangle$ cannot decay faster than by powers of $t$, asymptotically for $t \rightarrow \infty$.

This peculiar property is very well documented for the case of the 1D $s=\frac{1}{2}$ anisotropic $X Y$ model

$\mathscr{H}=-\sum_{l=1}^{N}\left[(1+\gamma) S_{l}^{x} S_{l+1}^{x}+(1-\gamma) S S^{y} Y_{+1}+h S_{l}^{z}\right]$,

whose dynamical properties have been analyzed in great detail. The pioneering work of McCoy, Barouch, and Abraham $^{32}$ on the general structure of the dynamic correlation functions of this model led to the remarkable conclusion that the time-dependent two-spin correlation functions $\left\langle S_{l}^{\mu}(t) S_{+R}^{\mu}\right\rangle$ for $T=0$ and fixed $R$ decay by powers of $t$ to their long-time asymptotic values irrespective of whether the corresponding equal-time correlation functions $\left\langle S_{l}^{\mu} S_{l_{+}}^{\mu}\right\rangle$ decay to their long-distance $(R \rightarrow \infty)$ asymptotic values exponentially in $R$ or by powers of $R .^{33}$

In the following, I should like to illustrate this charac- 
teristic long-time asymptotic behavior of two-spin correlation functions and the general structure of their excitation spectrum for two situations in the isotropic version of (3.9),

$$
\mathscr{H}=-\sum_{l=1}^{N}\left(S_{l}^{x} S_{l+1}^{x}+S Y S_{l+1}^{y}+h S_{l}^{z}\right) .
$$

The first situation $(h=1)$ corresponds to a state with maximum magnetic long-range order and the second situation $(h=0)$ to a state without any such order; the second situation represents a state with maximum spin fluctuations and the first situation a state without any correlated fluctuations.

At $h=1$ (first situation), the ground state of (3.9) is ferromagnetic with all spins aligned parallel to the magnetic field. This state is characterized by the following equaltime two-spin correlation functions:

$$
\begin{aligned}
& \left\langle S_{l}^{z} S_{l+R}^{z}\right\rangle=\left\langle S_{l}^{z}\right\rangle\left\langle S_{l+R}^{z}\right\rangle=\frac{1}{4}, \\
& \left\langle S_{l}^{x} S_{l+R}^{x}\right\rangle=\left\langle S_{l}^{y} S_{l+R}^{y}\right\rangle=\frac{1}{4} \delta_{R, 0} .
\end{aligned}
$$

The time-dependent autocorrelation functions, on the other hand, are readily determined to be

$$
\begin{aligned}
& \left\langle S_{l}^{z}(t) S_{l}^{z}\right\rangle=\left\langle S_{l}^{z}\right\rangle\left\langle S_{l}^{z}\right\rangle=\frac{1}{4}, \\
& \left\langle S_{l}^{x}(t) S_{l}^{x}\right\rangle=\frac{1}{4} e^{-i t} J_{0}(t),
\end{aligned}
$$

where $J_{0}(t)$ is a Bessel function. Both functions exhibit statistical behavior. The first is a constant and the second decays to zero for $t \rightarrow \infty$. The leading term of the longtime asymptotic expansion is

$$
\left\langle S_{l}^{x}(t) S_{l}^{x}\right\rangle \sim 2^{-5 / 2} \pi^{-1 / 2}(i t)^{-1 / 2}\left(1+e^{2 i(t+1 / 2)}\right) .
$$

The difference in behavior of the two functions (3.11) arises from the fact that the ground state is an eigenstate of the operator $S_{l}^{z}$ but not of the operator $S_{l}^{x}$. Therefore $S_{l}^{z}$ does not couple to any states other than the ground state itself, whereas $S_{l}^{x}$ couples to the branch of spin-wave excitations with dispersion

$$
\omega(q)=1-\cos q \text {. }
$$

As is well known, the $X Y$ model maps, via the JordanWigner transformation, ${ }^{34,35}$

$$
S_{l}^{+} \equiv S_{l}^{x}+i S_{l}^{y}=a_{l}^{\dagger} \exp \left(i \pi \sum_{j<l} a_{j}^{\dagger} a_{j}\right),
$$

$$
\begin{aligned}
& \phi_{z z}(\omega)=\frac{4}{\pi} F\left[\arcsin \left(\left\{\frac{1}{2}\left[1-\left(1-\omega^{2}\right)^{1 / 2}\right]\right\}^{1 / 2} / k_{1}\right), k_{2}\right] \Theta \\
& k_{1}=\left[1-(\omega / 4)^{2}\right]^{1 / 2}, \quad k_{2}=\left[1-(\omega / 2)^{2}\right]^{1 / 2}
\end{aligned}
$$

The ground state at $h=0$ corresponds to a half-filled Fermi sea, and the operator $S_{l}^{z},(3.14 c)$ couples to the particle-hole excitations. These excitations form a twoparameter continuum with upper and lower boundaries given by ${ }^{39}$

$$
\epsilon_{U}(q)=2\left|\sin \frac{q}{2}\right|, \quad \epsilon_{L}(q)=|\sin q|,
$$

$$
\begin{aligned}
& S_{l}^{-} \equiv S_{l}^{x}-i S_{l}^{y}=\exp \left[-i \pi \sum_{j<l} a_{j}^{\dagger} a_{j}\right] a_{l}, \\
& S_{l}^{z}=a_{l}^{\dagger} a_{l}-\frac{1}{2},
\end{aligned}
$$

onto a system of noninteracting fermions with the following one-particle spectrum:

$$
\omega_{k}=h+\cos k \text {. }
$$

In the fermion language the ground state for $h=1$ is the empty Fermi sea and the spin-wave excitations are essentially one-fermion states. All this is reflected in the frequency-dependent autocorrelation function $\phi_{\mu \mu}(\omega)$ defined in (3.7). The Fourier transforms of (3.11) are

$$
\begin{aligned}
& \phi_{z z}(\omega)=\frac{\pi}{2} \delta(\omega), \\
& \phi_{x x}(\omega)=\frac{1}{2\left[1-(1-\omega)^{2}\right]^{1 / 2}} .
\end{aligned}
$$

The square-root divergences in (3.16b) at $\omega=0$ and 2 obviously reflect the van Hove singularities of the spin-wave density of states.

At $h=0$ (second situation), the ground state of (3.9) corresponds to a $T_{c}=0$ critical point with no magnetic long-range order. The equal-time two-spin correlation functions decay as powers of $R$ asymptotically for large distances, ${ }^{34,36,37}$

$$
\begin{aligned}
& \left\langle S_{l}^{z} S_{l+R}^{z}\right\rangle \sim R^{-2}, \\
& \left\langle S_{l}^{x} S_{l+R}^{x}\right\rangle \sim R^{-1 / 2} .
\end{aligned}
$$

According to $(3.14 \mathrm{c})$, the time-dependent autocorrelation function $\left\langle S_{l}^{z}(t) S_{l}^{z}\right\rangle$ can be expressed as a density-density correlation function of noninteracting fermions. ${ }^{37}$ The result for $T=0,{ }^{27}$

$$
\left\langle S_{l}^{z}(t) S_{l}^{z}\right\rangle=\frac{1}{4}\left[J_{0}(t)+i E_{0}(t)\right]^{2},
$$

is again consistent with statistical behavior. $E_{0}(t)$ is a Weber function. The leading term in the long-time asymptotic expansion is

$$
\left\langle S_{l}^{z}(t) S_{l}^{z}\right\rangle \sim \frac{1}{2 \pi} e^{-2 i t}(-i t)^{-1}
$$

The frequency-dependent Fourier transform of (3.18) can be expressed in terms of elliptic integrals as follows: $:^{38}$ respectively. Since all matrix elements $\left\langle G\left|S_{q}^{z}\right| \lambda\right\rangle$ for the operator

$$
S_{q}^{z}=N^{-1 / 2} \sum_{l=1}^{N} e^{i q l} S_{l}^{z}
$$

between the ground state $|G\rangle$ and the particle-hole excitations $|\lambda\rangle$ have the same value, ${ }^{39}$ 


$$
\left|\left\langle G\left|S_{q}^{z}\right| \lambda\right\rangle\right|^{2}=2 / N
$$

the autocorrelation function $\phi_{z z}(\omega)$ given in (3.20) is, in fact, proportional to the density of particle-hole excitations. It has finite (nondivergent) van Hove singularities at $\omega=0,1,2$ respectively, of the following form: ${ }^{40}$

$$
\phi_{z z}(\omega) \sim\left\{\begin{array}{l}
\frac{2}{\pi} \omega \Theta(\omega) \\
-2^{5 / 2} \pi^{-1}(1-\omega)^{1 / 2} \Theta(1-\omega) \\
\Theta(2-\omega) .
\end{array}\right.
$$

The autocorrelation functions $\phi_{x x}(\omega)=\phi_{y y}(\omega)$, in contrast, have a much more complicated structure in the fermion representation. As a result of the exponential operators in the Jordan-Wigner transformation (3.14), they involve not just two-particle excitations as is the case for $\phi_{z z}(\omega)$, but rather the excitation of arbitrarily many particles. This fact was first established by McCoy, Barouch, and Abraham, ${ }^{32}$ who found that the time-dependent twospin correlation function $\left\langle S_{l}^{x}(t) S_{l+R}^{x}\right\rangle$ can be expressed in terms of an infinite block Töplitz determinant. The structure of the autocorrelation function $(R=0)$ and its frequency-dependent Fourier transform was recently analyzed in great detail. ${ }^{38,41,42}$ The long-time asymptotic expansion of $\left\langle S_{l}^{x}(t) S_{l}^{x}\right\rangle$ was found to have the following structure: ${ }^{38}$

$$
\begin{aligned}
& \left\langle S_{l}^{x}(t) S_{l}^{x}\right\rangle \sim 2^{-3 / 2} A^{2}(i t)^{-1 / 2} \sum_{m=0}^{\infty} T_{m}, \\
& T_{m}=(2 \pi)^{-m / 2} e^{-i m t}(-i t)^{-\beta_{m}} \sum_{n=0}^{\infty} b_{n}^{(m)}(-i t)^{-n},
\end{aligned}
$$

where

$$
A=2^{1 / 12} \exp \left[3 \xi^{\prime}(-1)\right]=0.64500248 \ldots
$$

and the coefficients $b_{n}^{(m)}$ are positive rational numbers. The exponents $\beta_{m}$ are given by

$$
\beta_{m}=\frac{1}{2}\left[\frac{1}{2}\left(m^{2}+1\right)\right] \text {, }
$$

where $[v]$ denotes the integer part of $v$, and $\zeta(z)$ is the Riemann zeta function.

In agreement with the requirement of statistical behavior this function decays to zero asymptotically for long times. The long-time asymptotic expansion (3.25) consists of an infinite sum of terms $T_{m}, m=0,1,2, \ldots$ each with a specific oscillatory $t$ dependence given by the phase factor $e^{-i m t}$. Each term $T_{m}$, which has been called a tower, is itself an infinite sum of terms with ascending powers of $t^{-1}$.

Quite generally, the long-time asymptotic expansion of $\left\langle S^{\mu}(t) S^{\mu}\right\rangle$ determines the singularity structure of its frequency-dependent counterpart $\phi_{\mu \mu}(\omega)$. Specifically, each tower $T_{m}$ of (3.25b) determines the singularity of $\phi_{x x}(\omega)$ at frequency $\omega=m$. The dominant contribution for each $m$ is the following: ${ }^{38}$

$$
\left.\phi_{x x}(\omega)\right|_{\omega \simeq m} \sim\left\{\begin{array}{l}
\frac{1}{2} B_{m} \Gamma\left(\frac{1}{2}-\frac{1}{4} m^{2}\right)(\omega-m)^{v_{m}} \Theta(\omega-m), \text { even } m \\
-\frac{1}{2} B_{m} \frac{1}{\left[\frac{1}{4}\left(m^{2}-1\right)\right] !}|\omega-m|^{v_{m}} \ln |\omega-m|, \text { odd } m
\end{array}\right.
$$

where

$$
B_{m}=A^{2} \sqrt{2}(2 \pi)^{-m / 2} b_{0}^{(m)}
$$

and $v_{m}=\frac{1}{4}\left(m^{2}-2\right)$ for even $m$ and $v_{m}=\frac{1}{4}\left(m^{2}-1\right)$ for odd $m$. The characteristic properties of the results (3.25) and (3.28), which are likely to reflect many generic features of the dynamical properties of integrable quantum many-body systems, may be summarized as follows.

(i) The function $\left[\phi_{x x}(\omega)\right]_{h=0}$ has nonzero spectral weight for arbitrarily high frequencies. In contrast, the functions $\left[\phi_{x x}(\omega)\right]_{h=1}$, Eq. (3.16b), and $\left[\phi_{z z}(\omega)\right]_{h=0}$, Eq. (3.20), are both of compact support.

(ii) The function $\left[\phi_{x x}(\omega)\right]_{h=0}$ has an infinite set of singularities at frequencies $\omega=m, m=0,1,2, \ldots$ of the form (3.28). These singularities are alternatingly onesided power type ( $m$ even) and two-sided power type with logarithmic corrections ( $m$ odd). In contrast, the function $\left[\phi_{x x}(\omega)\right]_{h=1}$ has just two singularities and the function $\left[\phi_{z z}(\omega)\right]_{h=0}$ has three. This is reflected in the fact that the long-time asymptotic expansions of the corresponding time-dependent autocorrelation functions, Eqs. (3.11b) and (3.18), involve only two and three towers, respectively, as opposed to the infinite series of towers in (3.25).

(iii) The regular pattern of singularities in $\left[\phi_{x x}(\omega)\right]_{h=0}$ is attributable to the fact that the excitation spectrum can be decomposed (in the fermion language) into sets of $m$ particle excitations with energies

$$
\epsilon_{m}\left(k_{1}, k_{2}, \ldots, k_{m}\right)=\sum_{l=1}^{m}\left|\omega_{k_{l}}\right|, \quad q=\sum_{l=1}^{m} k_{l},
$$

and $m$ arbitrarily large, whose densities of states have van Hove singularities at exactly the frequencies $\omega=m$, $m=0,1,2, \ldots$ However, the exact nature (exponents, amplitudes) of the singularities as they appear in $\left[\phi_{x x}(\omega)\right]_{h=0}$ is governed by the effects of matrix elements. The two singularities of $\left[\phi_{x x}(\omega)\right]_{h=1}$ are due to the oneparticle excitations of the empty Fermi sea and the three singularities of $\left[\phi_{z z}(\omega)\right]_{h=0}$ are due to the two-particle excitations of the half-filled Fermi sea.

(iv) It is noteworthy that the function $\left[\phi_{x x}(\omega)\right]_{h=0}$ can be represented (by means of a coordinate transformation in spin space) into a density-density correlation function of interacting fermions. The coupling to the $m$-particle excitations for $m>2$ can then be understood as being caused by the infinite hierarchy of Green's functions generated in the equation of motion for the two-particle Green's function by the interaction term of the fermion Hamiltonian. 
In summary, we observe that the time-dependent autocorrelation functions for the ground state of this integrable quantum-spin model (3.9) decay by powers of $t$ to their asymptotic values under vastly different circumstances. For a finite spin chain of length $N$, the number of excitations contributing to $\left[\phi_{x x}(\omega)\right]_{h=1}$ is $O(N)$, for $\left[\phi_{z z}(\omega)\right]_{h=0}$ it is $O\left(N^{2}\right)$ and for $\left[\phi_{x x}(\omega)\right]_{h=0}$ it is $O\left(2^{N-1}\right)$, out of a total of $2^{N}$ eigenstates.

All existing exact results point to the following conclusion: The constraint that time-dependent two-spin correlation functions for pure quantum states $\left\langle\psi\left|S_{l}^{\mu}(t) S_{+R}^{\mu}\right| \psi\right\rangle$ have a power-law long-time asymptotic behavior is as much a universal feature of integrable quantum-spin systems in the thermodynamic limit $N \rightarrow \infty$ as the constraint that all trajectories are confined to $N$-dimensional hypersurfaces in the $2 N$-dimensional phase space is a universal feature of integrable classical spin clusters. In the classical system, the constraint is the consequence of the fact that the entire phase space is foliated by invariant tori, whereas in the quantum system it is the consequence of the fact that the entire excitation spectrum has a multiparameter continuum structure. This strongly suggests that a characteristic property of nonintegrable quantum-spin models might be the following: there exist pure quantum states $|\psi\rangle$ (not necessarily eigenstates of $\mathscr{H}$ ) for which the time-dependent autocorrelation function $\left\langle\psi\left|S_{l}^{\mu}(t) S_{l}^{\mu}\right| \psi\right\rangle$ decays more rapidly to its asymptotic value than a power law (possibly exponentially). This effect of nonintegrability would parallel the well-known result that exponentially decaying autocorrelation functions occur in nonintegrable classical dynamical systems with few degrees of freedom. ${ }^{43,44}$ This has far-reaching consequences.

An exponentially decaying autocorrelation function $\left.\langle\psi \mid S\rangle^{\mu}(t) S\right|^{\mu}|\psi\rangle$ implies the existence of new classes of excitations with qualitatively different spectral properties, spectra without (power-law or logarithmic) van Hove singularities in their densities of states. Nonintegrable quantum-spin systems would then possess two types of spectrum, the regular spectrum and the irregular spectrum in analogy to the two types of spectrum of a nonintegrable classical spin system: the discrete spectrum associated with regular trajectories and the continuous spectrum associated with chaotic trajectories. For the quantum many-body system, the two types of spectrum may be characterized as follows.

(i) Regular spectrum. This part of the spectrum consists of multiparameter continua. It is characterized by the property that for increasing $N$ individual excitations belonging to a particular branch or continuum close up in $(\mathbf{q}, \omega)$ space as $1 / N$ to an increasingly regular pattern. This property was verified by exact calculations on integrable $1 D$ quantum-spin models ${ }^{39}$ and is, in fact, the very basis for the usefulness of finite-chain calculations for the determination of spectral properties and $T=0$ dynamic structure factors of $1 D$ quantum-spin models. The regular spectrum generally exists also in nonintegrable quantum-spin models. For example, in a large class of $1 D$ spin- $s$ models with ferromagnetic ground state, most of which are believed to be nonintegrable, the regular spectrum includes the classes of excitations with one over- turned spin (spin waves) and two overturned spins (twospin-wave continuum and branch of bound spin complexes). ${ }^{45}$ These branches and continua of excitations play a role similar to that of invariant tori for the classical spin clusters. However, the spectrum of a quantum-invariant torus is, in general, not discrete but continuous unlike the spectrum of its classical counterpart, the reason being that the quantum many-body system has an infinite number of degrees of freedom. It is the regular multiparameter continuum structure which is the hallmark of a quantuminvariant torus. Every excitation belonging to the regular spectrum can be characterized unambiguously in terms of a set of quantum numbers. In integrable spin models all excitations belong to this class. Selection rules play a major role in the regular spectrum. For example, the particle-hole excitations contributing to $\left[\phi_{z z}(\omega)\right]_{h=0}, \mathrm{Eq}$. (3.20), comprise only a small subset of those excitations which are not already excluded by selection rules associated with global symmetry properties of the Hamiltonian (3.9). ${ }^{46}$

(ii) Irregular spectrum. In nonintegrable quantum-spin models there also exist excitations which do not form regular patterns with a multiparameter continuum structure in the thermodynamic limit. For finite $N$, this part of the spectrum is likely to share many characteristics of the irregular spectrum observed in quantum-spin clusters for large $s$ (as described in Sec. III A). However, in contrast to the case of a spin cluster in the classical limit $s \rightarrow \infty$, the irregular spectrum of a quantum-spin model in the thermodynamic limit $N \rightarrow \infty$ is not describable by a piecewise smooth energy density with isolated singularities. The absence in the irregular spectrum of individual branches or continua with well defined boundaries, which would invariably lead to van Hove singularities, must be the result of strong level repulsion, which in turn is attributable to the nonexistence of a sufficient number of conservation laws (expressible in terms of a complete set of quantum numbers). It is highly suggestive to view in these new classes of excitations manifestations of quantum chaos.

Thus far we have pointed out the similarities and differences between quantum integrability (the theme of Sec. III) and classical dynamical integrability (the theme of Sec. II A). However, it is important to realize that quantum integrability is also closely related to the thermodynamic integrability of classical (many-body) statistical models (the theme of Sec. II B). This relation is established by the well-known exact mappings between Hamiltonians of 1D quantum-spin models and transfer operators of certain 2D classical statistical models or, more generally, between $D$-dimensional quantum models and $(D+1)$ dimensional classical models. ${ }^{47,48}$ Some of the characteristic features of nonintegrability in quantum manybody systems (i.e., features of quantum chaos) as postulated in this paper can then be translated into corresponding effects of thermodynamic nonintegrability in classical statistical models. ${ }^{49}$

In summary, the preceding discussion has naturally led to the conjecture that quantum chaos in spin systems is an intrinsic long-time asymptotic property of nonintegrable quantum systems in the thermodynamic limit $N \rightarrow \infty$. 
This view of the nature of quantum chaos deviates distinctively from the two views paraphrased at the beginning of this paper. In contrast to classical dynamical chaos, which makes its appearance in systems with as few as two degrees of freedom, quantum chaos requires an infinite number of degrees of freedom, and is therefore much more difficult to analyze theoretically. ${ }^{50}$ In practice, the study of quantum chaos is limited for the most part to the study of its precursors in finite- $N$ systems of nonintegrable quantum many-body models.

At present, the available evidence for nonintegrability effects in 1D quantum-spin models which may be attributable to quantum chaos is still somewhat circumstantial. Systematic investigations are in progress. Two pieces of evidence are briefly outlined in the following.

(i) Finite-chain extrapolations for various thermodynamic quantities of 1D quantum-spin models have been successfully tested on the integrable $s=\frac{1}{2} X Y$ model (3.9). However, recent attempts to determine the staggered susceptibility $\chi_{x x}(q=\pi)$ at $T=0$ by means of finite-chain extrapolations have dramatically failed in an unprecedented way. ${ }^{51,52} \chi_{x x}(\pi)$ indeed represents the linear response to a perturbation which renders the model nonintegrable. The exact result for $\chi_{x x}(\pi)$, by which the finite-chain results could be tested, was determined from the exactly known time-dependent correlation functions of the unperturbed, i.e., integrable model. ${ }^{53}$

(ii) A number of unusual and puzzling features have recently been observed in the ground-state and spectral properties of the 1D $s>\frac{1}{2}$ Heisenberg antiferromagnet (3.2), which is believed to be nonintegrable in contrast to its Bethe-ansatz-solvable $s=\frac{1}{2}$ counterpart. Various numerical studies of this model, which have been motivated by Haldane's ${ }^{54}$ challenging prediction (based to a large ex- tent on approximate mappings to continuum models) that the integer- $s$ Heisenberg antiferromagnetic chains have an energy gap in the excitation spectrum while the halfinteger-spin counterparts do not, have resulted in a state of controversy: Finite-chain extrapolations are inconclusive due to erratic behavior and slow convergence; $55-58$ this makes finite-size scaling quite unreliable. Also the results of different Monte Carlo studies are in disagreement over important issues. ${ }^{59,60}$ It is not surprising that the phenomenon of level repulsion which is expected in the irregular spectrum of a nonintegrable model makes finite-chain extrapolations extremely difficult if not impossible. In the absence of a complete set of quantum numbers by which every eigenstate can be uniquely identified, the tracking of a particular excitation in systems of increasing $N$ is an ambiguous process. Changes in trend occurring at various states of finite-chain extrapolations have indeed been observed in supposedly nonintegrable quantum-spin chains. ${ }^{61}$

\section{ACKNOWLEDGMENTS}

I am indebted to B. M. McCoy for several discussions on nonintegrability in classical statistical models, which were inspirational for the present work. Furthermore, I have greately benefited from collaborative projects with $J$. C. Bonner and R. E. Shrock, which proved to be instrumental for many insights reported in this work. I should like to thank also C. Kaufman, M. C. Gutzwiller, E. Magyari, M. P. Nightingale, and H. Thomas for numerous interesting and very useful discussions. This work was supported in part by the Council on Research of the University of Rhode Island and by the U.S. National Science Foundation, Grant No. DMR-86-03036.
${ }^{1}$ Chaotic Behavior in Quantum Systems. Theory and Applications, edited by G. Casati (Plenum, New York, 1985).

2Since each classical spin corresponds to one mechanical degree of freedom (as explained in Sec. II A), $N$ is at the same time the number of spins.

${ }^{3}$ A. J. Lichtenberg and M. A. Lieberman, Regular and Stochastic Motion (Springer, New York, 1983).

${ }^{4} \mathrm{~A}$ more technical definition of thermodynamic integrability such as the applicability of the Bethe ansatz or the existence of a nontrivial solution of the Yang-Baxter relations is likely to be too restrictive.

${ }^{5}$ R. J. Baxter, Exactly Solved Models in Statistical Mechanics (Academic, New York, 1982).

${ }^{6}$ It is noteworthy that the Poisson brackets (2.5) can also be inferred if the $S_{l}$ are interpreted as classical angular momentum variables $S_{l}=Q_{l} \times P_{l}$ in terms of $3 N$ position and momentum variables $Q t^{\mu}$ and $P \mu, \mu=x, y, z$, respectively. However, it is important to realize that the classical spin has no mechanical analog in terms of a rotating rigid body (for example, a microscopic top) with equivalent dynamical properties. The inertial tensor of a classical spin would have to be characterized by a single nonvanishing principal moment, which is "unmechanical."
${ }^{7}$ Since $\theta_{l}, \phi_{l}$ are angular variables, the underlying phase space is actually a $2 N$-dimensional compact manifold.

${ }^{8}$ The set of frequencies is, in general, no longer finite if the transformation from the spin variables to the action-angle variables is nonlinear.

${ }^{9}$ K. Nakamura, Y. Nakahara, and A. R. Bishop, Phys. Rev. Lett. 54, 861 (1985).

${ }^{10}$ E. Magyari, H. Thomas, R. Weber, C. Kaufman, and G. Müller, Z. Phys. B (to be published).

${ }^{11}$ M. Feingold and A. Peres, Physica 9D, 433 (1983).

${ }^{12}$ M. Feingold, N. Moiseyev, and A. Peres, Phys. Rev. A 30, 509 (1984).

${ }^{13}$ H. Frahm and H. J. Mikeska, Z. Phys. B 60, 117 (1985).

${ }^{14}$ Quite generally, a nonautonomous classical Hamiltonian system with $N$ degrees of freedom can be transformed into an autonomous system with $N+1$ degrees of freedom (see Ref. 3 for details).

${ }^{15}$ It seems that thermodynamic nonintegrability in 1D spin models cannot arise except, perhaps, in the presence of infinite-range interactions.

${ }^{16}$ H. A. Kramers and G. H. Wannier, Phys. Rev. 60, 252 (1941); T. Nakamura, J. Phys. Soc. Jpn. 7, 264 (1952); M. E. Fisher, Am. J. Phys. 32, 343 (1964); G. S. Joyce, Phys. Rev. Lett. 19, 
581 (1967); Phys. Rev. 155, 478 (1967); H. E. Stanley, ibid. 179, 570 (1969); L. L. Liu and R. J. Joseph, Phys. Rev. Lett. 26, 1378 (1971).

${ }^{17}$ B. M. McCoy, (unpublished); B. M. McCoy and M. L. Yan, Nucl. Phys. B257 [ FS14], 303 (1985).

${ }^{18}$ This statement is subject to some qualifications, which were pointed out by J. D. Johnson, S. Krinsky, and B. M. McCoy, Phys. Rev. A 8, 2526 (1973).

${ }^{19}$ Very similar observations were made on small quantum systems of quite different nature (see, e.g., Ref. 1).

${ }^{20}$ A. Peres, Ref. 1, p. 165.

${ }^{21}$ For example, the parameters $A$ or $J$ in (2.15) and the parameters $h$ or $J$ in (2.16).

${ }^{22}$ N. G. van Kampen, Ref. 1, p. 309.

${ }^{23}$ The observation that these precursors of classical dynamical chaos in quantum-spin clusters arise not only in timedependent (dynamical) properties but to some extent also in the quantum energy spectrum can be understood by the fact (discussed previously) that there is only one all-encompassing concept of integrability in quantum systems. Hence, precursors of classical dynamical chaos in quantum-spin clusters turn into true chaos in the classical limit $s \rightarrow \infty$ if they are observed in dynamical quantities such as the time evolution of Wigner distributions, but fade away ultimately if they are observed in "static" quantities such as the distribution function of energy levels. No such dichotomy exists in the other limit where nonintegrability effects make their appearance: $N \rightarrow \infty$ with $s$ finite (see Sec. III B).

${ }^{24}$ H. A. Bethe, Z. Phys. 71, 205 (1931).

${ }^{25}$ R. Orbach, Phys. Rev. 112, 309 (1958); C. N. Yang and C. P. Yang, ibid. 150, 321 (1966); 150, 327 (1966); 151, 258 (1966); J. Des Cloizeaux and M. Gaudin, J. Math. Phys. 7, 1384 (1966).

${ }^{26}$ B. Sutherland, Phys. Rev. B 12, 3795 (1975); L. A. Takhtajan, Phys. Lett. 87A, 479 (1982); H. M. Babujian, ibid. 90A, 479 (1982).

${ }^{27}$ S. Katsura, T. Horiguchi, and M. Suzuki, Physica 46, 67 (1976).

28J. H. H. Perk, H. W. Capel, and T. J. Siskens, Physica 89A, 304 (1977).

${ }^{29}$ R. V. Jensen and R. Shankar, Phys. Rev. Lett. 54, 1879 (1985).

${ }^{30}$ Isolated dispersion branches are one-parameter continua. Two-parameter continua of excitations in 1D quantum-spin systems are discussed extensively, e.g., in Ref. 39 below.

${ }^{31}$ This event is not known to occur in any dynamic correlation function of a pure quantum state. However, the structure of time-dependent correlation functions is qualitatively different for $T>0$; see, e.g., H. W. Capel and J. H. H. Perk, Physica 87A, 211 (1977); J. M. R. Roldan, B. M. McCoy, and J. H. H. Perk, Physica 136A, 255 (1986).

${ }^{32}$ B. M. McCoy, E. Barouch, and D. B. Abraham, Phys. Rev. A 4, 2331 (1971)

${ }^{33}$ The only exceptions pertain to very special circumstances for which the time-dependent correlation functions are either constant, periodic, or multiperiodic functions.

${ }^{34}$ E. Lieb, T. Schultz, and D. Mattis, Ann. Phys. (N.Y.) 16, 407 (1961).

${ }^{35}$ S. Katsura, Phys. Rev. 127, 1508 (1962).

${ }^{36}$ B. M. McCoy, Phys. Rev. 173, 531 (1968).

${ }^{37}$ T. Niemeijer, Physica 36, 377 (1967).

${ }^{38}$ G. Müller and R. E. Shrock, Phys. Rev. Lett. 51, 219 (1983); Phys. Rev. B 29, 288 (1984).

${ }^{39}$ G. Müller, H. Thomas, H. Beck, and J. C. Bonner, Phys. Rev.
B 24, 1429 (1981).

${ }^{40}$ See G. Müller and R. E. Shrock, Phys. Rev. B 29, 288 (1984), Fig. 5 for plots of both the two-parameter continuum of particle-hole excitations and the corresponding density of states.

${ }^{41}$ H. G. Vaidya and C. A. Tracy, Physica 92A, 1 (1978).

${ }^{42}$ B. M. McCoy, J. H. H. Perk, and R. E. Shrock, Nucl. Phys. B220 [FS8], 35 (1983); 220 [FS8] 269 (1983).

${ }^{43}$ G. M. Zaslavsky, Chaos in Dynamic Systems (Harwood, New York, 1985).

${ }^{44}$ It is similar to the effect observed by Frahm and Mikeska (Ref. 13) in the nonautonomous one-spin model (2.17) near the classical limit, except for the important difference that in their case it reflects a precursor of classical dynamical chaos, whereas in the context of infinite quantum-spin systems it would be attributable to true quantum chaos. Their observation that the width of the wave function of a Wigner distribution corresponding to a chaotic trajectory in the classical limit increases (roughly) exponentially with time also implies an exponential decay of the corresponding spin autocorrelation function.

${ }^{45}$ R. P. Hodgson and J. B. Parkinson, J. Phys. C 18, 6385 (1985).

${ }^{46}$ The rotational symmetry of the $X Y$ Hamiltonian allows only excitations with quantum number $S_{T}^{z}=0$ to contribute to $\left[\phi_{x x}(\omega)\right]_{h=0}$, given the fact that the ground state at $h=0$ has also quantum number $S_{T}^{z}=0$.

${ }^{47}$ M. Suzuki, Prog. Theor. Phys. 46, 1337 (1971); 56, 1454 (1976).

${ }^{48}$ Special cases of the integrable 1D $s=\frac{1}{2} X Y Z$ model, for example, map onto the exactly solved $2 \mathrm{D}$ Ising, six-vertex and eight-vertex models (see Ref. 5).

${ }^{49}$ Suppose we have a nonintegrable 1D quantum-spin system whose ground state couples to the irregular spectrum of that model (not necessarily exclusively). If the situation corresponds to the critical point of a $T_{c}=0$ phase transition (which commonly occur in 1D quantum-spin systems), the irregular spectrum is likely to upset the standard scaling behavior, which so heavily depends on the infinite quasidegeneracy of energy levels adjacent to the ground state as discussed in Sec. III B, i.e., on the multiparameter continuum structure of the spectrum in the vicinity of the ground state. Translated into properties of the transfer operator of the associated 2D classical statistical model this would imply similar consequences for the critical behavior of nonintegrable classical statistical models.

${ }^{50}$ Whereas the numerical integration of nonintegrable classical dynamical systems is a relatively straightforward procedure, the determination of the time evolution of nonintegrable (i.e., infinite) quantum systems is a formidable task even for short times.

${ }^{51}$ P. M. Duxbury, J. Oitmaa, M. N. Barber, A. van der Bilt, K. O. Joung, and R. L. Carlin, Phys. Rev. B 24, 5149 (1981).

52J. C. Bonner, One Dimensional Systems, Vol. C 140, of Lecture Notes for NATO ASI Summer School on Magnetostructural Correlations in Exchange Coupled Systems, edited by R. D. Willett, D. Gatteschi, and O. Kahn (Reidel, Dortrecht, 1985), p. 157.

${ }^{53}$ G. Müller and R. E. Shrock, Phys. Rev. B 30, 5254 (1984); 31, 637 (1985).

${ }^{54}$ F. D. M. Haldane, Phys. Lett. 93A, 464 (1983); Phys. Rev. Lett. 50, 1153 (1983).

${ }^{55}$ R. Botet and R. Jullien, Phys. Rev. B 27, 618 (1983).

56J. C. Bonner and G. Müller, Phys. Rev. B 29, 5216 (1984). 
57U. Glaus and T. Schneider, Phys. Rev. B 30, 215 (1984).

58J. B. Parkinson, J. C. Bonner, G. Müller, M. P. Nightingale, and H. W. J. Blöte, J. Appl. Phys. 57, 3319 (1985); J. C. Bonner, G. Müller, M. P. Nightingale, H. W. J. Blöte, and J. B. Parkinson, J. Magn. Magn. Mat. 54, 1253 (1986).
${ }^{59}$ M. P. Nightingale and H. W. J. Blöte, Phys. Rev. B 33, 659 (1986).

${ }^{60} \mathrm{~K}$. Sogo and M. Uchinami, J. Phys. A 19, 493 (1986).

61 J. Oitmaa, J. B. Parkinson, and J. C. Bonner, J. Phys. C (to be published). 